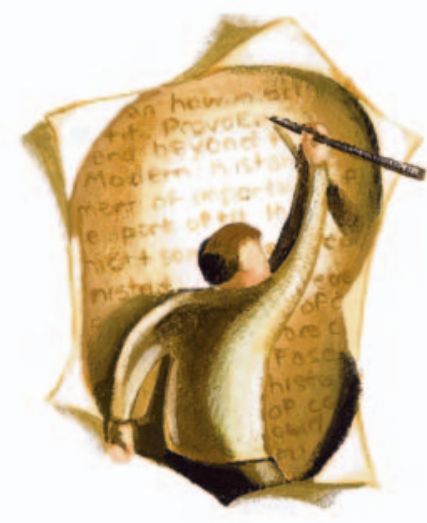

\section{$\beta$-Blockers for hypertension}

Nadia Khan and Finlay McAlister ${ }^{1}$ conclude that $\beta$-blockers are efficacious for hypertension in younger but not in older patients, but their conclusions are based on questionable statistical methods.

In their Methods section, Khan and McAlister state that "Meta-analyses for all outcomes were performed using random-effects models." When I tried to reproduce their results by entering data into Review Manager (the Cochrane Collaboration's software for conducting reviews; version 4.2 for Windows), I also observed a significant reduction of cardiovascular events in younger patients (relative risk [RR] $0.86,95 \%$ confidence interval [CI] 0.74-0.99), but this result was based on a fixed-effects model. With the "true" random-effects model, the $\mathrm{CI}$ was wider and included the value I (RR 0.86, 95\% CI 0.75-I.00). Relative to other antihypertensive drugs, $\beta$-blockers seemed to increase the risk of cardiovascular events in older patients (RR I.06, 95\% CI I.0I-I.Io), but again in a fixed-effects model. With a randomeffects model, the CI includes I (RR I.07, 95\% CI I.00-I.I4).

\section{Falk Hoffmann}

Drug Utilization Research Unit International University Bremen Bremen, Germany

\section{REFERENCE}

I. Khan N, McAlister FA. Re-examining the efficacy of $\beta$-blockers for the treatment of hypertension: a meta-analysis. CMAJ 2006;174(I2):1737-42.

DOI:Io.1503/cmaj.106org8
Nadia Khan and Finlay McAlister ${ }^{1}$ defined the primary outcome for their meta-analysis as the composite cardiovascular outcome of death, nonfatal myocardial infaraction or nonfatal stroke. Unfortunately, this definition is unclear; as a result, for some studies only cardiovascular deaths and for other studies all-cause deaths were included in the analyses. We checked the values presented for all trials in Figs. $2 \mathrm{~A}$ and $2 \mathrm{~B}$ against the original publications and found several discrepancies (see Appendix I available at www.cmaj.ca/cgi/content/full/176/7 197I-a/DCI). We recommend recalculating the results and rethinking the interpretation.

\section{Andrea Siebenhofer \\ Consultant Physician \\ Karl Horvath \\ Consultant Physician \\ Klaus Jeitler \\ Thomas Gratzer \\ Medical University Graz \\ Graz, Austria}

\section{REFERENCE}

I. Khan N, McAlister FA. Re-examining the efficacy of $\beta$-blockers for the treatment of hypertension: a meta-analysis. CMAJ 2006;174(I2):1737-42.

DOI:Io.I503/cmaj.ro6org9

Nadia Khan and Finlay McAlister ${ }^{1}$ reexamined our meta-analysis ${ }^{2}$ of $\beta$ blockers in primary hypertension but came to a different conclusion than we did. We would like to clarify why the conclusions differ.

First, we examined the effect of $\beta$ blocker treatment on the incidence of myocardial infarction (MI), stroke or death separately, whereas Khan and McAlister focused on the composite end point of all 3 conditions. However, antihypertensive drugs do not have the same relative effect on stroke incidence as on MI or death.

Second, we excluded the results of the Captopril Prevention Project (CAPPP) trial, ${ }^{3}$ because it is impossible to retrieve data on how many patients in that study were receiving $\beta$-blockers. ${ }^{2}$ CAPPP had a PROBE design (prospective, randomized, open treatment with blinded end-point evaluation), ${ }^{3}$ as well as some other major quality concerns; for example, randomization was imbalanced, with more high-risk patients receiving captopril than conventional treatment (diuretics and/or $\beta$-blockers), and suboptimal use of captopril once daily was encouraged in an unknown number of patients. There is no way of extrapolating from other Scandinavian trials the percentage of patients in the CAPPP study who were treated with $\beta$-blockers, since both investigators and patients differed among these trials.

Finally, cardiovascular outcome after treatment of primary hypertension in subjects under 60 years of age is poorly documented. Therefore $\beta$ blockers cannot be recommended for any age group.

\section{Bo C. Carlberg \\ Department of Medicine \\ University Hospital \\ Umeå, Sweden}

\section{REFERENCES}

I. Khan N, McAlister FA. Re-examining the efficacy of $\beta$-blockers for the treatment of hypertension: a meta-analysis. CMAJ 2006;174(I2):1737-42.

2. Lindholm LH, Carlberg B, Samuelsson O. Should $\beta$-blockers remain first choice in the treatment of primary hypertension? A meta-analysis. Lancet 2005;366:1545-53.

3. Hansson L, Lindholm LH, Niskanen L, et al. Effect of angiotensin-converting-enzyme inhibition compared with conventional therapy on cardiovascular morbidity and mortality in hypertension: the Captopril Prevention Project (CAPPP) randomised trial. Lancet $1999 ; 353: 6 \mathrm{II}-6$.

DOI:I0.I503/cmaj.Io60205

\section{[The authors respond:]}

Falk Hoffman asserts that the conclusions in our meta-analysis were incorrect, as the random-effects confidence intervals for Figs. IA and $2 \mathrm{~B}$ appeared to include the value $\mathrm{I}$ when the analyses were repeated with Review Manager software. However, unlike Hoffman, we conducted our random-effects analyses using a soft- 
ware program that does not round off at 2 decimal places. Thus, in both random- and fixed-effects modelling, the confidence intervals approached but did not incorporate $\mathrm{I}$. The summary effect estimate for the comparison in Fig. IA ( $\beta$-blocker v. placebo in trials enrolling younger patients) with the random-effects model is 0.862 (95\% CI $0.746-0.996 ; p=$ 0.044 ); This result (for which the 95\% CI in our original publication was truncated, rather than rounded, to $0.74^{-0.99)}$ clearly supports the interpretation stated in our paper that $\beta$-blockers are more efficacious than placebo in younger patients. Similarly, the summary effect estimate for the comparison in Fig. 2B ( $\beta$-blocker $v$. other antihypertensives in trials enrolling older patients) with the random-effects model is 1.066 (95\% CI I.00I-I.I35, incorrectly reported in our original publication as 1.06 with 95\% CI I.OI-I.IO; $p=0.047$ ); this result supports our interpretation that $\beta$-blockers were less efficacious than other antihypertensive agents in older patients. Unfortunately, this estimate and $95 \%$ CI were incorrectly reported in our paper; in rounded form, these values should have been reported as I.07 (95\% CI I.OI-I.IO). ${ }^{1}$

Andrea Siebenhofer and colleagues note that our definition of the composite outcome was unclear. The initial version of our meta-analysis was substantially longer than the final published version and thus contained far more detail on trial entry criteria, baseline characteristics and outcome definitions. For publication purposes, we were asked to shorten the manuscript, and we regret that in doing so we inadvertently caused confusion for some readers. To clarify, our primary analyses were for the composite outcome of cardiovascular deaths (or all-cause deaths where cardiovascular deaths were not reported), MI or stroke. In light of the concerns of Siebenhofer and colleagues about the $\mathrm{I} 2$ trials included in Figs. $2 \mathrm{~A}$ and $2 \mathrm{~B}$ of our meta-analysis, it is important to point out that the composite outcomes incorporated cardiovascular mortality in 9 of those trials, total mortality in 2 and cardiac mortality in $\mathrm{I}$. Ten of the $\mathrm{I} 2$ trials incorporated fatal and nonfatal MI and stroke in their composite outcomes (one trial included only fatal and nonfatal MI and another included only fatal MI and stroke). We did not have access to the primary study data and therefore relied on the end-point definitions and methods of classification used by the primary trialists (recognizing that this was consistent within trials and thus would be identical across treatment groups within each trial).

In their table, Siebenhofer and colleagues report a different number of events in the composite outcome for the CAPPP trial than we did. Although we defined the composite event rates as the proportion of patients experiencing any of the composite events (and thus counted each participant only once), Sienbenhofer and colleagues appear to have pooled the number of events for the individual end points separately to arrive at their composite event rates. However, this individual counting of events assumes that they are independent and that no patients suffered more than one event; this is clearly not the case and we therefore favour our approach (which was that taken by the CAPPP authors in their primary publication $^{2}$ ).

Siebenhofer and colleagues and Bo Carlberg questioned our inclusion of data from CAPPP and STOP2 (mixed $\beta$ blocker studies). We discussed the reasons for including these trials in our paper, ${ }^{1}$ where we also reported 2 sensitivity analyses that address these concerns.

Finally, Carlberg points out that the meta-analysis that he and his colleagues conducted differed from ours in that they examined the effect of $\beta$ blocker treatment on the incidence of MI, stroke or death separately and based their conclusions on the excess risk of stroke observed in patients treated with $\beta$-blockers. On the other hand, we focused on the composite end point of all 3 conditions together to account for competing risks and the potential for survivor bias. As discussed in our paper, an agent with a beneficial effect on one end point may appear to have a detrimental effect on another end point, given that treated patients survive without the first end point for long enough that the second end point occurs.

\section{Nadia Khan}

Division of General Internal Medicine University of British Columbia

Vancouver, BC

\section{Finlay McAlister}

Division of General Internal Medicine University of Alberta

Edmonton, Alta.

\section{REFERENCES}

I. Khan N, McAlister FA. Re-examining the efficacy of $\beta$-blockers for the treatment of hypertension: a meta-analysis. CMAJ 2006;174(12):1737-42.

2. Hansson L, Lindholm LH, Niskanen L, et al. Effect of angiotensin-converting-enzyme inhibition compared with conventional therapy on cardiovascular morbidity and mortality in hypertension: the Captopril Prevention Project (CAPPP) randomised trial. Lancet I999;353:6II-6.

DOI:I0.I503/cmaj.I060200

\section{Coombs' testing}

\section{and neonatal}

\section{hyperbilirubinemia}

Although we fully agree with Michael Sgro and colleagues regarding the need for early identification and effective management of neonatal hyperbilirubinemia, we were surprised to see their recommendation that the Coombs' test be used to screen for hyperbilirubinemia in all infants born to mothers with type O blood. ${ }^{1}$ Although the Coombs' or direct antibody test (DAT) is an important test when trying to identify the cause of neonatal hyperbilirubinemia, recent studies have shown that it has extremely limited usefulness in predicting the development of significant hyperbilirubinemia.

The DAT has been shown to have a positive predictive value of $12 \%-53 \%$ and a sensitivity of $15 \%-64 \%$ for the subsequent development of hyperbilirubinemia, which limits its usefulness as a screening test (Table I). ${ }^{2-4}$ Herschel and colleagues found that in comparison with measurements of end-tidal carbon monoxide concentration, the DAT showed only $8.5 \%$ sensi- 